\title{
Percepción paterna acerca de su participación en el parto
}

\author{
Fathers' perception about their participation in childbirth
}

\section{Ana María Silva Dreyer ${ }^{1}$ Cynthia Carrasco Carré2 ${ }^{2}$ Daniela Oliva Sánchez ${ }^{3}$, Catalina González Salas ${ }^{3}$, Ángela Gálvez Loyola ${ }^{3}$, Flavia Faúndez López ${ }^{3}$, Bárbara Tapia Jofré ${ }^{3}$, Ariadna Orrego Vergara ${ }^{3}$, Yuseska Cid Armijo ${ }^{3}$}

${ }^{1}$ Escuela de Obstetricia y Puericultura, Universidad de Valparaíso. Centro Interdisciplinario de Estudios en Salud, Universidad de Valparaíso. Centro Interdisciplinario de Investigación en Salud Territorial, Campus San Felipe, Universidad de Valparaíso. La Troya esquina El Convento s/n. San Felipe

${ }^{2}$ Escuela de Obstetricia y Puericultura, Universidad de Valparaíso. Centro Interdisciplinario de Estudios en Salud, Universidad de Valparaíso. Angamos 655, Reñaca, Viña del Mar.

${ }^{3}$ Escuela de Obstetricia y Puericultura, Universidad de Valparaíso. La Troya esquina El Convento s/n. San Felipe

*Autor para correspondencia: anamaria.silva@uv.cl

RECIBIDO: 14 de Diciembre de 2020

APROBADO: 29 de Abril de 2021

DOI: $10.22370 /$ rev.mat.2.2021.2533

ESTUDIO DERIVADO DE PROCESO DE TESIS DE PREGRADO | LOS AUTORES DECLARAN NO TENER CONFLICTO DE INTERESES

Palabras claves: Parto, Participación padre, Percepción.

Key words: Birth delivery, Father participation, Perception.

\section{RESUMEN}

Antecedentes: La atención del parto se ha ido modificando progresivamente, dando paso a una atención personalizada, la cual releva el acompañamiento a la mujer. En Chile hay avances en la materia, aun cuando los estudios suelen estar más centrados en el efecto en la mujer que en la vivencia de los padres. Conocer su visión es relevante para la promoción de su participación.

Objetivo: Describir la percepción que tienen los padres acerca de su participación en el parto.

Material y método: Estudio cualitativo, que aplicó una entrevista semiestructurada a 28 participantes. La información fue procesada mediante análisis de contenido cualitativo.

Resultados: La percepción general de los padres acerca de su participación en el parto es positiva, y saben que es un derecho. Una motivación central es considerar especial el momento, en el cual deben estar presentes. Valoran haber sido partícipes, destacan haber podido apoyar a sus parejas, y opinan haber recibido una buena atención por parte del equipo de salud. No obstante, para algunos fue una experiencia negativa por el sufrimiento de la madre, y por insatisfacción con la atención clínica recibida. Cierta insatisfacción se dio por la resolución de tipo de parto vía cesárea, a pesar de la preferencia por un parto natural.

Conclusiones: Los padres valoran su participación en el parto como experiencia relevante en sus vidas. Se observan condiciones favorables para involucrarlos más activamente. Se recomienda mayor educación antenatal, participación en la decisión del tipo de parto, adecuación física hospitalaria, y desarrollo de facilidades y legislación laboral.

ABSTRACT
Background: Delivery care has been progressively
modified towards a personalized health care model,
that considers accompaniment of the mother in this
process as relevant. In Chile there are advances in
the matter, even though studies tend to be more fo-


cused on the women s wellbeing than on the father's experience. Knowing their vision is relevant to promote their participation.

Objective: Describe fatheŕ s perception about their participation in childbirth.

Methods: Qualitative study, which applied a semi-structured interview to 28 participants. The information was processes through qualitative content analysis.

Results: The general perception of fathers about their participation in childbirth is positive, and they know that it is their right. A central motivation is to consider the moment special, in which they must be present. They value their participation, highlight having been able to support their partner, and believe to have been well treated by the health team. However, for some fathers it was a negative experience due to the mother's suffering and dissatisfaction with the clinical care received. Some dissatisfaction arose from the resolution of the type of delivery via cesarean section, despite the preference for a natural delivery.

\section{INTRODUCCIÓN}

El nacimiento constituye una de las etapas del parto y consiste en la expulsión o extracción del feto desde el cuerpo materno. Es un proceso íntimo para la gestante, altamente sensible (1), dado lo cual en las últimas décadas se han desarrollado esfuerzos tendientes a que sea una experiencia positiva. En Chile, a partir del año 2008 se ha promovido una modalidad de atención del parto denominada "personalizada" (2), acorde a recomendaciones de organismos internacionales (3)(4)(5). Se expresa en un manual (2) y en orientaciones de la red de protección de la infancia Chile Crece Contigo (6). Entre las recomendaciones se encuentra la de acompañamiento de la madre por parte de una persona significativa, preferentemente del padre, cuando éste es una figura presente y ambos lo deseen.

En estudios primarios y revisiones de literatura, se señala que el acompañamiento del progenitor en la gestación y el parto tiene efectos positivos en la relación emocional del padre con la madre y el recién nacido, aumentando el bienestar materno durante el parto (7)(8)(9)(10)(11) y aunque no incide en la mortalidad, mejora los resultados obstétricos (12) (13). En Chile, investigaciones han llegado a conclusiones similares. Para las mujeres la presencia del padre es valorada (14)(15)(16)(17)(18)(19), y se ha observado más participación paterna en los cuidados del recién nacido cuando es activamente involucrado en las actividades del parto por parte del equipo de salud (18).

La implementación de las recomendaciones de parto personalizado en Chile ha dado lugar a estudios evaluativos, que evidencian diversos obstáculos. En cuanto al acompañamiento, si bien se reportan avances, aún se observa una brecha (14)(15)(19)(20) (21), a raíz de diversas limitaciones y exclusiones de índole cultural, institucional y social para la participación paterna (14)(15), lo que también ha sido estudiado en otros países, como España (21).

Si bien el rol del padre en este proceso de gestación y parto ha sido declarado relevante, es aún limitado el conocimiento que existe respecto a la visión o perspectiva del progenitor sobre el parto y sobre su participación en éste (22). Los estudios suelen centrarse y recoger información sobre la percepción de las mujeres gestantes o puérperas, y los varones son relegados a roles secundarios del proceso. Algunos trabajos, sin embargo, han evidenciado que los padres valoran su participación en el parto (14)(15) (20)(21). Tal como se indica en las orientaciones para la implementación del modelo de atención integral en salud (23) y en la declaración de Alma-Ata (24), se favorece la participación de los sujetos en las diversas acciones sanitarias, en un modelo que se centra en la persona y en su perspectiva, basada en sus conocimientos, experiencias y creencias. Sin embargo, para lograr su mayor involucramiento, es necesario conocer su visión acerca de los temas en los cuales han de participar.

El objetivo del presente estudio es describir la percepción de padres residentes en la comuna de San Felipe, acerca de su participación en el proceso de parto. La finalidad de la investigación es contribuir a la integración del padre en el periodo reproductivo, mediante el aporte de información procedente directamente de los sujetos involucrados, los padres, que permita implementar estrategias efectivas que estimulen y faciliten su participación. 


\section{MATERIAL Y MÉTODO}

Lugar de estudio: El estudio tuvo lugar en la comuna de San Felipe, Región de Valparaíso, durante el año 2019.

Diseño: La investigación fue de enfoque cualitativo, dado el objetivo de conocer la vivencia y percepciones de los padres presentes en el proceso del parto (25)(26)(27)(28). El estudio fue de alcance descriptivo, exploratorio, debido a que actualmente en nuestro país hay escasa evidencia acerca de la perspectiva paterna.

Participantes: La muestra se conformó acorde el criterio de diversidad en términos de edad, nivel educacional, ocupación, y participación en uno o más de un parto (ver distribución en tabla 1 de resultados) y la estrategia de bola de nieve (también denominado de redes o cadena), a partir del contacto inicial que pudieron establecer con algunos de ellos los miembros del equipo de investigación (26)(27). Se incluyeron personas mayores de edad, de nacionalidad chilena, residentes en San Felipe, que fueron padres en los últimos tres años. Se excluyeron padres que hubiesen tenido una experiencia compleja en relación con el embarazo o el parto. La muestra resultante consistió en veintiocho participantes, cuyas respuestas saturaron información relativa a los temas centrales del estudio.

Variables: Las variables del estudio, asociadas a los objetivos específicos y que guiaron la pauta de entrevista, son: motivación de los progenitores para participar en el parto; experiencia de participación del padre en éste; propuestas de los progenitores para su mayor incorporación en el proceso (Anexo 1 dimensiones y subdimensiones).

Recolección de datos: Se recurrió a la entrevista semiestructurada, basada en una pauta temática flexible en su aplicación, grabada con el consentimiento informado del participante, la cual fue previamente piloteada, y revisada por dos matrones varones, con experiencia paterna de participación en el parto. El lugar de la realización de las entrevistas fueron sitios convenidos con los participantes. El estudio fue aprobado por el Comité de Ética del Servicio de Salud Aconcagua, se rigió por las normas éticas vigentes y de acuerdo con la Asociación Médica Mundial y la Declaración de Helsinki.
Análisis de datos. Los datos obtenidos de las entrevistas fueron procesados mediante Análisis de Contenido cualitativo narrativo (26)(27)(29)(30)(31). Las entrevistas audio grabadas fueron transcritas literalmente. El texto resultante fue fragmentado en unidades de sentido para cada entrevista, las que fueron codificadas, asignándoles una etiqueta (código) que diera cuenta de los datos. Este proceso fue realizado por dos miembros del equipo de investigación, bajo la conducción y revisión posterior de la investigadora principal. Luego se procedió a agrupar los códigos del conjunto de entrevistas en categorías temáticas, en torno a las cuales se describieron los resultados integrados de las entrevistas.

\section{RESULTADOS}

\section{Participantes}

Los padres entrevistados, acorde a los criterios de selección, fueron diversos en sus características sociodemográficas.

\section{Tabla 1}

Características de los veintiocho participantes

\begin{tabular}{llc}
\hline Características & Alternativas & $\begin{array}{c}\text { Número de } \\
\text { Entrevistados }\end{array}$ \\
Edad & Mayores de 35 años & 15 \\
& $\begin{array}{l}\text { Menores de 35 } \\
\text { años }\end{array}$ & 13 \\
Escolaridad & Básica & 1 \\
& Media & 11 \\
Ocupación & Superior & 15 \\
& $\begin{array}{l}\text { Trabajador } \\
\text { dependiente }\end{array}$ & 21 \\
& $\begin{array}{l}\text { Trabajador } \\
\text { independiente }\end{array}$ & 3 \\
& Estudiante & 16 \\
Veces que ha & Primera ocasión & 4 \\
& $\begin{array}{l}\text { Segunda ocasión } \\
\text { o más }\end{array}$ & 12 \\
\hline
\end{tabular}




\section{Motivación e incertidumbres iniciales}

Deseo de participación: La totalidad de los entrevistados quería participar en el parto de su hijo o hija, por considerarlo un momento especial y relevante, para apoyar a la pareja, y para generar vínculos con ésta y el recién nacido.

"Asistir era algo importante que me iba a marcar, que iba a tener el recuerdo el resto de mi vida" (BT3P14).

Algunas de las causas por las que otros padres no participen, serían la falta de tiempo, poca voluntad, timidez, o temor. Se señala que es algo que no hay que forzar.

Expectativas y sentimientos previos al parto: Para un grupo se trató de un primer hijo/a y por tanto experiencia nueva. Sentimientos expresados fueron nerviosismo, ansiedad, expectación. También temor a complicaciones, al sufrimiento materno, a eventuales falencias médicas, a la sangre y a desmayarse, y desconfianza hacia los hospitales.

"Se tenía el miedo de que pudiera ocurrir cualquier estupidez de lo que ha sucedido a lo largo de todo el país, el cambio de hijo, la no preocupación, la falencia que hay de repente en los servicios" (CG4P7).

\section{Vivencia de participación en el parto}

Facilidades para la participación paterna: La mayoría de los padres entrevistados tuvo facilidades para ingresar a preparto, parto, postparto y neonatología, tanto en la clínica, como en el hospital. Solo algunos tuvieron restricciones para ingresar a la sala de ecografía durante la gestación. Uno de ellos, que no pudo ingresar a la sala de parto, vivió la situación con temor.

“...Estuvo sola todo ese rato, incluso escuchaba gritos $y$ con mucha incertidumbre de saber qué pasaba, yo de repente golpeaba y no salía nadie" (YC1P9).

Rol: Los entrevistados opinan que su rol principal fue el apoyo, la compañía, la demostración de cariño y la contención a la pareja. Si bien a algunos padres les habría gustado participar más, la conformidad con este rol limitado se asocia al parto como escenario médico, sobre todo en caso de una cesárea.

"Sí, yo le hablaba, le ayudaba a respirar, la hacía calmarse. Le contaba sobre otras cosas. Igual veía que cuando yo le hablaba, ella se sentía mejor" (YC4P15).

Preparto: Menos de la mitad de los padres señalan haber acompañado a sus parejas a los controles prenatales. Aunque ya estando en el hospital, la mayoría en algún momento pudo acompañar a la madre en la etapa de preparto.

"Estuve con mi señora todo el proceso anterior, estuve con ella cuando entró al parto y me dejaron con mi bebé después de que naciera" (YC4P1).

Algunos, sin embargo, no pudieron entrar al preparto, sino tan solo al momento del expulsivo, o cuando sus hijos ya habían nacido. Dentro de las razones por las cuales no entraron, se menciona que no se les permitió. Un padre no sabía, y tampoco preguntó, asumió que era así.

“...... Ella fue ingresada y yo traté de entrar y me abrieron la puerta un segundo, y me dijeron "está en preparto, espere" y me cerraron la puerta" (FF1P1).

Parto: De manera generalizada, los padres entrevistados tuvieron una percepción positiva de su asistencia al parto.

"Más que agradable, fue como emocionante. Fue bien bonito en realidad" (DO3P15).

En la experiencia positiva influyó la atención recibida, otros se refieren al apoyo y vínculo con la pareja, otros a la importancia de saber qué acontecía y cómo estaba la madre.

No obstante, pueden también emerger sentimientos encontrados, más negativos: varios de ellos mencionan el nerviosismo y la preocupación por la pareja, la observación del sufrimiento de la madre, o lo traumático que fue presenciar una cesárea. 
"Tan desagradable que yo en un momento dije que el motivo de no querer tener otro hijo es por el sufrimiento que pasó ella" (AG2P4).

El alumbramiento. Respecto al momento del alumbramiento, se destaca la emoción al ver nacer a sus hijos y al tener a su hijo o hija en los brazos por primera vez.

"Fue mucho mejor de lo que yo pensaba, ver nacer al Benjamin" (DO2P9).

Postparto: Muchos de los entrevistados pudieron presenciar la atención inmediata de sus hijos, aunque el nivel de participación en ésta fue más bien bajo. Solo a unos pocos se les permitió participar activamente.

"Eh, claro, me hicieron, me llevaron a ver cómo la estaban midiendo, circunferencia de cabeza, peso, en eso uno participa, claro. En la clínica, aquí no" (CG3P17).

\section{Conocimientos previos}

Derecho a participar: Cerca de la mitad de las personas entrevistadas no sabía que el acompañamiento a la madre en el parto es un derecho, o desconocía detalles de éste.

"No tenía idea que era un derecho de uno" (BT4P40).

"Sabía que en el momento del parto sí podía estar, pero en el pre y post, no estoy seguro" (FF4P18).

La principal fuente de información fueron los controles prenatales. Otras fuentes de información mencionadas fueron una asistente social, redes sociales, internet, televisión, revistas, experiencia anterior; y en dos casos, la formación profesional (matrones).

Socialización entre pares. La gran mayoría de los entrevistados conoce a otros padres que participaron en el parto. Señalan que entre amigos han compartido acerca del hecho como experiencia vital y también desconocido para los hombres, e intercam- biado información acerca de los procedimientos. Sin embargo, también se menciona que no es el tipo de cosas que se comparte mucho entre hombres.

"Para los hombres es un mundo desconocido, porque pertenece como al mundo de la mujer" (AO2P6).

"No son como cosas que uno generalmente comparte entre hombres, como que las conversaciones son más superficiales habitualmente... a no ser que alguien se sienta tan mal que necesite desahogarse" (FF3P14).

\section{Tipo de parto}

Si bien el foco del estudio fue la participación paterna en el parto, de las entrevistas emergió el tema del tipo de parto, las preferencias de los padres en torno a éste, y su participación.

Tipo de parto: En relación con el tipo de parto al que asistieron, en la mayoría de los casos fue operación cesárea. De éstos, solo en tres casos fue por una decisión personal de la pareja.

Preferencias: Solo cuatro padres preferían la cesárea ya desde antes del parto, por temor a complicaciones, porque la madre lo prefiere o por mala vivencia de un parto anterior. La mayoría que prefería parto vaginal mencionó como razón el deseo de la madre de vivir un parto natural, y la más rápida recuperación de la madre.

La cesárea como limitante a la participación: Los padres que asistieron a un parto por cesárea asumieron como normal su menor participación en el momento del alumbramiento, por considerarse un acto quirúrgico.

\section{Interacción con los servicios de salud}

Lugar del parto: Casi todos los padres entrevistados señalan que el parto ocurrió en un hospital público. Tan solo tres padres asistieron a una clínica.

Experiencia general e interacción con el personal de salud: La experiencia general positiva por parte de la mayoría de los participantes se asocia al buen trato, a la información recibida, a la preocupación y acompañamiento por parte de los profesiona- 
les de la salud, así como de los estudiantes de obstetricia. La confianza fue mayor cuando los padres ya conocían al personal médico y matrona desde antes.

“...Fue super agradable, nos sentimos super acompañados en todo momento, desde que llegó al preparto por la matrona, anestesista, gineco obstetras, pediatra en la atención inmediata, fue algo super agradable super acompañado $y \mathrm{hu-}$ mano, hacia mi y a mi pareja" (FF4P11).

Un padre asocia el buen trato de los profesionales de salud con la elevada tolerancia al dolor de la pareja.

"Tampoco tuve ningún inconveniente, mi senora nunca ha sido una persona que sea buena para quejarse. Eso ayuda a que el personal del hospital la tomara de otra forma, y a que a uno le faciliten las cosas..." (CG4P15).

Algunos padres, no obstante, encontraron negativa la experiencia por una atención clínica considerada deficiente y falta de contención emocional por parte del personal de salud.

"Mira, la verdad que, con la matrona, que es la que llegó por entre comillas por urgencia, fue un trato muy desagradable, horrible... Yo estuve a punto de no sé, pelear con ella" (AG2P7).

"Si hay algo malo, es el hospital, que no sean tan negligentes. Imagínese a mi señora no se dieron cuenta que la niña venía atravesada..." (YC1P8).

Información recibida: Cerca de la mitad de los entrevistados recibió información en todo momento sobre los procedimientos que se realizaban.

"Las matronas muy buenas, te aconsejan mucho, te dejan muy tranquilo; te explica todo lo que está pasando, lo que hace" (AG4P13).

Sin embargo, se encuentran críticas acerca de esta información recibida, por considerarla insuficiente.

"Sí, faltó que me dieran más información, que yo no sabía que estaba pasando... Mi señora después me dijo que como las 5 de la mañana rompió bolsa y a mi no me contaron nada..." (YC1P12).
"Que te dieran una instrucción, dónde pararte, dónde ir, cosas así. Y que el profesional esté instruido a entender el estado emocional que está uno, explicarle mejor, hacerlo participar" (AG1P12).

\section{Propuestas para aumentar o facilitar la par- ticipación paterna}

Información y educación: En general los entrevistados opinan que se debe aumentar la educación acerca del parto y su proceso físico, la vivencia materna, y los procedimientos. También de cómo contener a la pareja, así como la relevancia y beneficios de la participación paterna.

Otra propuesta es compartir la vivencia entre pares, para motivar a los padres a participar, para que no sientan tanto temor.

"Mmm, no sé, tratar de transmitirle mi punto de vista, que es grato po, que es una experiencia inolvidable" (AG1P17).

"Que no se dejen llevar por los comentarios de los demás, que hagan lo que sienten. Yo creo que falta que no metan tanto miedo, que no es algo terrible" (DO4P36).

Atención integral y humanizada: Hay quien plantea el deseo de una atención integral del proceso de parto, que trascienda el hacer "lo justo y necesario" para obtener un buen resultado de salud perinatal.

"De que trataran mejor a la mujer. Yo hacia el símil de como de, guardando la proporción, de un matadero, como que la función solo es ir para allá y expulsar la guagua y que esté bien y que no se muera, ¿cachai? No es cercana la interacción profesional/mujer" (AG1P16).

Se proponen acciones como aumentar el tiempo y participación del padre en el preparto, darle mayor libertad de movimiento a la mujer, promover mayor interacción entre la pareja y que el equipo intervenga menos, dejar que el padre sea parte de la experiencia y exprese sus emociones, preocuparse de detalles, como entregar un vaso de agua o un calmante al padre. 
Trato al usuario: Si bien la percepción de trato al usuario en general es positiva, hay quienes entre las propuestas se refieren a la necesidad de mejorarlo en el conjunto del servicio.

“...Yo sé que los del servicio de salud están cansados, estresados, pero los malos tratos no se justifican .... Deberían ponerse en el lugar del familiar que está sufriendo o asustado. No sólo hablo del parto, sino en todo el servicio de salud" (YC2P7).

También se plantea que debiese existir un mecanismo para regular las negligencias médicas.

"Pero tienen que haber mecanismos para regular que se actúe dentro de la práctica médica y eso noté yo que faltó" (FF2P77).

Infraestructura y equipamiento: Se plantea que la dotación actual no facilita la participación paterna. Se propone un pabellón exclusivo de maternidad que permita la presencia del padre, salas de parto personalizado, mayor separación entre las camas en preparto. una sala de espera acondicionada con asientos y baño para los padres.

"Sería ideal contar con salas de parto personalizado, donde pudiesen estar los padres acompañando en todo momento a la mujer, porque las camas de preparto están muy juntas. Porque además al lado hay otra persona que le están haciendo procedimientos y el padre tiene que salir" (AO2P17).

Institucionalización de procesos: A nivel institucional se propone crear un protocolo que permita una experiencia integral, que indique paso a paso las acciones a realizar y que se dé a conocer a madres y padres ya desde el nivel primario de atención.

Evolución social y cultural: Entre las propuestas de cambio a nivel societal, se señala la necesidad de desarrollar la legislación laboral para facilitar la participación paterna en el todo el proceso de parto, así como en los controles prenatales y el apoyo post natal.
"Que existiera una ley que ayude a que uno pueda ausentarse por ciertas cantidades de horas del trabajo para poder seguir el proceso completo" (CG4P19).

De la narrativa emerge otra dimensión social: se asocia un mejor trato y mayor participación paterna en el parto, a la atención privada de salud, no así con la pública. Del mismo modo, se asocia la utilización del bono de pago asociado a diagnóstico (PAD) de FONASA, que permite libre elección del médico, acceso a servicios privados y a una mayor calidad de la atención.

"Fue súper agradable. La matrona era la que estaba haciendo rondas más seguido. Ella nos preguntaba cómo estábamos, si nos faltaba algo. Pero claro, también era porque estábamos en una clínica" (AO4P21).

"Y como se quedó en pensionado, podía estar todo el tiempo con ella y mi hijo" (DO3P20).

En el ámbito cultural, se considera necesario cambiar la cultura considerada machista, en la cual el padre queda relegado del proceso crianza. Se hace un llamado a los padres a involucrarse más y a las mujeres a incluir al progenitor tempranamente, desde la gestación.

"Yo creo que haciéndolo más participativo, del aspecto en que la mujer tiene que incluirlo, la mujer tiene que hacerlo participar en el proceso del embarazo, porque hay mujeres que no les gusta tampoco, y que la sociedad también saque esos paradigmas, que no, eso es solamente para la mujer" (CG3P22). 
El siguiente esquema sintetiza los resultados anteriormente detallados:

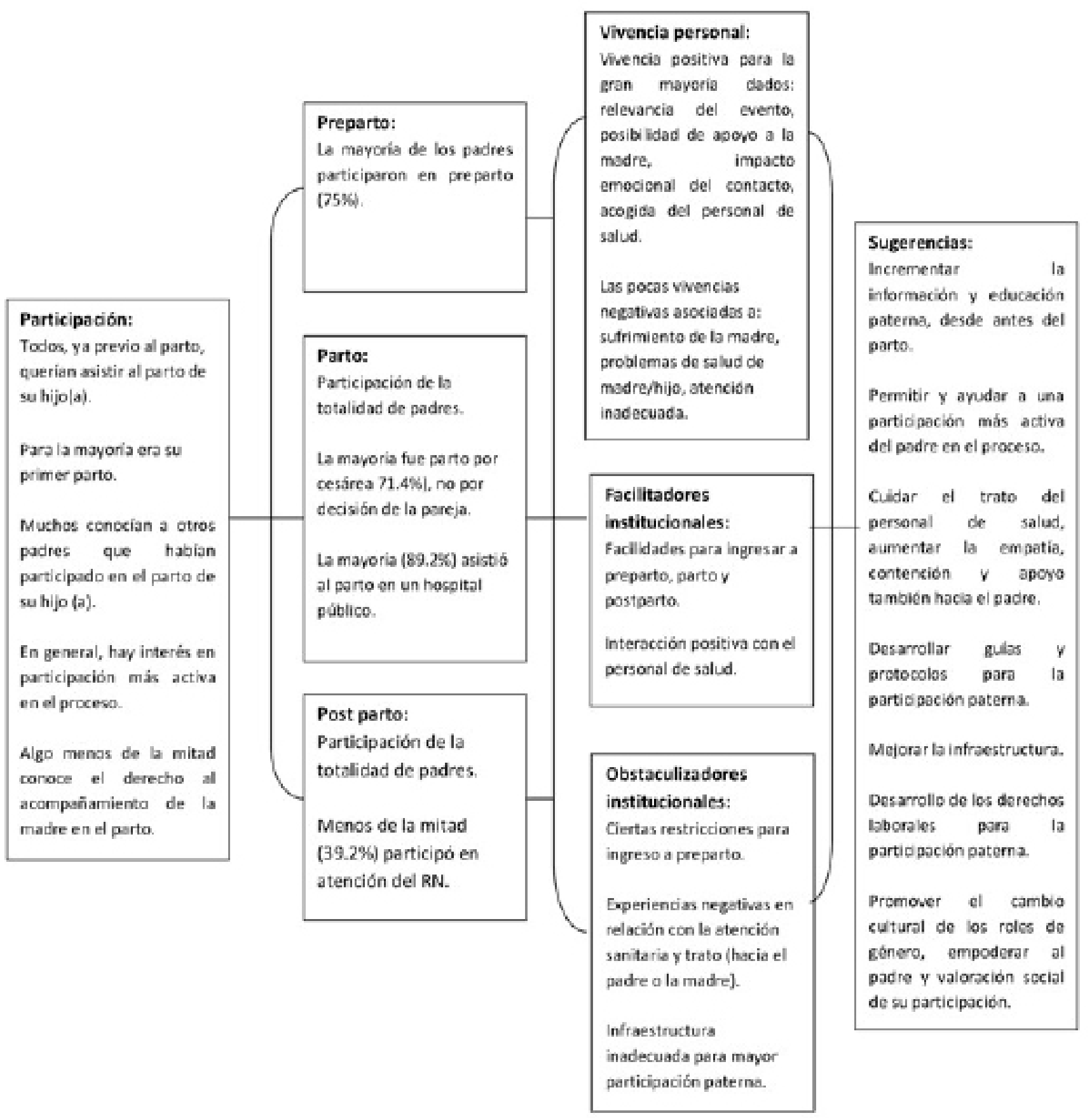




\section{DISCUSIÓN}

Como resultado central del estudio destaca la predisposición positiva de los padres de asistir al parto, ya desde la gestación. En una mirada retrospectiva, consideran que el nacimiento de un hijo o hija es un momento único y trascendente para un padre. Se refieren también a la relevancia de ser parte del proceso, de apoyar y de esta manera beneficiar a la madre, y de generar un vínculo precoz con el recién nacido. Esta tendencia positiva ha sido evidenciada en trabajos en diversos países, acorde a revisiones de literatura desarrolladas el año 2014 (7), 2015 (13) y 2016 (11). En Chile, ha sido documentada también por algunos estudios: un análisis cualitativo de testimonios paternos en el contexto de estudio experimental realizado en el Servicio de Salud Metropolitano Sur Oriente de Chile y una experiencia de promoción de participación paterna en el hospital regional de Coyhaique (17) (18), y una investigación antropológica acerca de la vivencia paterna en el parto (20).

Si bien la mayoría de los padres reporta la experiencia como positiva, algunos la recuerdan como vivencia negativa. Ésta se relaciona con el sufrimiento de la pareja, con un actuar médico a su juicio negligente o con tratos inadecuados del personal (médico, matrona) hacia ellos o sus parejas. Este tipo de queja ha sido recogida en un estudio nacional de evaluación de la aplicación de las recomendaciones ministeriales de parto personalizado en maternidades en Chile (19).

En general, los entrevistados tuvieron facilidades para asistir a todas las etapas del parto, principalmente a la de alumbramiento y refirió una interacción positiva con el personal de salud, quien salvo en algunos casos, les proporcionó información general acerca del proceso.

No obstante, les gustaría haber tenido un rol aún mayor, así como orientaciones más específicas y de aplicación práctica durante la gestación (poder participar en el control ecográfico, por ejemplo), el preparto y en la atención del recién nacido. Esto, en su opinión, debiera ser promovido, facilitado y guiado por el equipo de salud obstétrico, tal como ha sido planteado en otras investigaciones fuera (11) y dentro de Chile (18). Las afirmaciones anteriores se inscriben en la tendencia nacional de participación paterna creciente en las últimas décadas (19) (32). No obstante, todavía hay alrededor de un tercio o más de partos no acompañados (19), y se ha reportado (15) la menor importancia que los profesionales de salud le asignan a la interacción con los padres, en comparación con las madres, lo que también se observó en España (21).

Los entrevistados han planteado que sería conveniente que los padres compartan sus experiencias, para dar a conocer la relevancia del hecho entre pares, disminuir sus temores frente a un hecho que consideran parte del mundo de la mujer, y estar informados para poder solicitar y hacer efectiva su participación cuando ésta no sea considerada en los servicios de salud. Se señaló la necesidad de actualizar los protocolos y sensibilizar aún más a los equipos sanitarios para una mayor participación paterna, coincidiendo con las orientaciones ministeriales de parto personalizado (2) y participación paterna (6). Este esfuerzo incluye su preparación, pues hay padres a quienes no se les acurre, no desean, o tienen temor de asistir al parto (6).

Dado este rol del equipo de salud, se considera pertinente abordar estudios acerca de la percepción, motivaciones y propuestas del equipo de salud en los diferentes niveles de la red de salud, acerca de la participación paterna en el proceso de gestación, parto y puerperio. Esta brecha de información acerca de las expectativas paternas ha sido identificada en una revisión de literatura publicada recientemente (año 2018) en la revista Midwifery (22).

El obstáculo principal presentado por los entrevistados fue de índole laboral que limita los permisos para el acompañamiento paterno. Consideran que esta dificultad restringe su participación al no tener tiempo para asistir a controles de embarazo, parto, puerperio, y tener un rol más activo en la crianza temprana de sus hijos e hijas. Esta limitación fue documentada en el ya mencionado estudio de Aguayo (15). También señalaron como obstáculo, el factor cultural de menor participación paterna en la crianza, brecha de género que ha sido ya relevada en España (23). Un estudio cualitativo publicado por la Organización Panamericana de Salud (36) concluye en la contribución de la participación paterna activa en el parto en la aminoración de dicha brecha, en términos de que esta participación no disminuye su masculinidad. En el norte de América, los diversos obstáculos señalados han sido planteados, junto con estrategias para superarlos, por parte de una comisión destinada a investigar y proponer estrategias destinadas a fomentar la paternidad activa (37). 
Si bien no se trató del foco del estudio, emergió como resultado una elevada proporción de cesáreas, la cual es una limitante adicional a la participación paterna más activa, por tratarse de una intervención quirúrgica con mayores protocolos de seguridad. Esta elevada proporción de cesáreas sigue la tendencia nacional, la cual preocupa a diversos actores. El año 2017 el Colegio de Matronas expuso que, del total de los partos, el 43\% corresponde a cesáreas, cifra elevada en comparación al 31\% reportado en el año 2002 (33). Lo significativo en el estudio presente, es que la cesárea no responde a la preferencia de la pareja previa al parto: la mayoría prefería un parto vaginal a una cesárea, lo que coincide con una tendencia señalada ya en estudios de años anteriores en Chile (34) (35). Sería de interés estudiar las posibilidades de mayor participación paterna aún en contexto de cesárea mediante revisiones de literatura y estudios primarios que aborden la perspectiva tanto de los profesionales de salud como de los padres.

Como limitación de la presente investigación se puede señalar la baja cobertura territorial, consistente en una sola comuna del Valle de Aconcagua, la cual es a la vez predominantemente urbana (San Felipe, capital provincial). Sería de interés ampliar el trabajo e indagar en la percepción de padres del ámbito rural.

Una segunda limitación consiste en que la percepción en general positiva de los entrevistados, haya estado influida por su predisposición favorable a participar ya desde el momento de la gestación. Por ello, habría sido de interés la realización de grupos de discusión entre padres participantes, con el fin de profundizar en los resultados a partir de la interacción de los entrevistados. Por diversas limitaciones, no fue posible aplicar esta metodología en este estudio. Cabe señalar, sin embargo, que los resultados no son, en general, divergentes de algunos estudios realizados en otras regiones del país, como se detalló antes. Las preguntas por responder se relacionan con las motivaciones, percepciones, dificultades de padres que no han asistido al parto, tema de interés para ser abordado en investigaciones futuras, permitiendo una visión integrada en la materia. Como facilidad para la realización del estudio, destaca el interés de los padres en ser escuchados en el tema, así como su disposición de compartir su experiencia.

\section{CONCLUSIONES}

De los relatos de la experiencia paterna de asistencia al parto, se identifican condiciones propicias para promoverla, dadas las actitudes favorables mayoritarias observadas tanto en los padres como en el personal de salud. Los padres entrevistados, consideraron que su asistencia al parto fue un momento grato y significativo, en el cual destacan la importancia de su rol apoyando a su pareja, y de la vinculación temprana con el recién nacido. A la vez, salud frente al acompañamiento paterno.

No obstante, respecto de barreras que otros padres podrían tener para participar, la principal mencionada es la falta de tiempo y permisos asociados a su ocupación. Surge la propuesta de ampliar los derechos laborales para los padres, para así poder estar presentes en el todo el proceso y desde la gestación.

Como dificultades, también se mencionaron la falta de voluntad, temor o nerviosismo, y aspectos culturales que se expresan como brecha de género. Frente a ello, se propone involucrar más al padre desde el embarazo y proporcionarle educación acerca del parto y de la relevancia de su participación para la tríada. También se propuso que los establecimientos de salud deberían permitir la participación más activa, además de adecuar sus protocolos, infraestructura y equipamiento.

Con el fin de promover la participación paterna, y de superar las barreras, se considera necesario que el conjunto del equipo de salud (matrona, médico obstetra, técnicos de enfermería) y otros del servicio (por ejemplo, guardias de seguridad) disponga de conocimientos actualizados sobre la participación paterna, y que, en su ejercicio profesional, tanto en atención primaria como hospitalaria, facilite la integración del padre. Estas medidas, adicionalmente, son un aporte al cambio cultural. El interés paterno de mayor participación es una condición favorable para el cambio actitudinal tendiente a la equidad de género. 


\section{REFERENCIAS}

1. Velasco J. Aspectos emocionales que rodean el nacimiento. Estado de la cuestión. Matronas prof. 2005;6(4):23-7.

2. Ministerio de Salud. Manual de atención personalizada en el proceso reproductivo [Internet]. División Prevención y Control de Enfermedades. Subsecretaría de Salud Pública. 2008. 360 p. Available from: http://web.minsal.cl/portal/url/item/795c 688fd24acd68e04001011e013bfb.pdf

3. Organización Mundial de la Salud. Recomendaciones de la OMS para la conducción del trabajo del parto. 2015.

4. Organización Mundial de la Salud (OMS). Recomendaciones de la OMS Para los cuidados durante el parto, Transformar la atención a mujeres y neonatos para. Dep Salud Reprod e Investig Conex Organ Mund la Salud [Internet]. 2018;WHO-RHR-18(8):1-8. Available from: https://www.who.int/reproductivehealth/publications/intrapartum-care-guidelines/ es/

5. ICM IC of M. ICM Definitions [Internet]. [cited 2020 Dec 4]. Available from: https://www.internationalmidwives.org/our-work/policy-and-practice/icm-definitions.html

6. Forray Claps $\mathrm{C}$ (Chile CC. Un espacio novedoso para la Paternidad en Chile: La inclusión del padre en el embarazo, parto y cuidados del recién nacido. | Chile Crece Contigo [Internet]. [cited 2020 Dec 4]. Available from: http://www.crececontigo.gob. cl/columna/un-espacio-novedoso-para-la-paternidad-en-chile-la-inclusion-del-padre-en-el-embarazo-parto-y-cuidados-del-recien-nacido/

7. Ramírez H, Rodríguez I. Beneficios del acompañamiento a la mujer por parte de su pareja durante el embarazo, el parto y el puerperio en relación con el vínculo paternofilial. Matronas profesión [Internet]. 2014;15(4):1-6. Available from: https://www.federacion-matronas.org/wp-content/ uploads/2018/01/revision-beneficios-pareja.pdf

8. Rodriguez Gallego I, Ruiz Ferrón C, Partida Márquez AL. Evolution of Humanization Project on Perinatal Attention at the Universitary Hospital Virgen del Rocío of Sevilla. Nure Investig. 2016;13(83):116.

9. Melo RM de, Angelo BH de B, Pontes CM, Brito RS de. Men's knowledge of labor and childbirth. Esc Anna Nery - Rev Enferm. 2015;19(3):454-9.
10. Uribe C, Contreras A, Villarroel L. Trabajos Originales Adaptación y validación de la escala de bienestar materno en situación de parto: segunda versión para escenarios de asistencia integral. Rev Chil Obstet Ginecol [Internet]. 2014;79(3):154-60. Available from: $h t t p: / / w w w . s c i e l o . c l / s c i e l o . p h p ? s c r i p t=s c i$ arttextEpid=S0717-75262014000300002

11. Coutinho EC, Antunes JGVC, Duarte JC, Parreira VC, Chaves CMB, Nelas PAB. Benefits for the Father from their Involvement in the Labour and Birth Sequence. Procedia - Soc Behav Sci. 2016;217:43542.

12. Mincy R, Center for Research on Fathers, Children and FW. Overview: Paternal Involvement and Pregnancy Outcomes. Columbia University School of social Work NICHD Fatherhood Outreach Meeting, July 28-29, 2015.

13. Meier ME, Avillaneda L. A Literature Review of Paternal Involvement in Prenatal Care. J Fam Strengths [Internet]. 2015;15(1):10. Available from: http://digitalcommons.library.tmc.edu/jfs/vol15/ iss $1 / 10$

14. Aguayo F, Correa P, Cristi P. Resultados de la encuesta internacional de masculinidades y equidad de género. Caso chileno coordinado por CulturaSalud EME. 2011;32-49.

15. Aguayo F, Correa P. KE. Estudio de Paternidad en el Sistema Público de Salud CulturaSalud MINSAL. 2012:1-169.

16. Uribe C, Contreras A, Villarroel L, Rivera $\mathrm{S}$, Bravo P, Cornejo M. Bienestar materno durante el proceso de parto: Desarrollo y aplicación de una escala de Medición. Rev Chil Obstet Ginecol. 2008;73(1):4-10.

17. Uribe C, Contreras A, Hoga L. Presencia activa del padre en el nacimiento integral: significados atribuidos por padres y madres a los roles paternos. Rev Chil Obstet Ginecol. 2018;83(1):22-6.

18. Villalón HU, Toro RG, Riesco IC, Pinto MC, Silva C V. Participación paterna en la experiencia del parto). Rev Chil Pediatr. 2014;85(5):554-60.

19. Binfa L, Pantoja L, Ortiz J, Gurovich M, Cavada G, Foster J. Assessment of the implementation of the model of integrated and humanised midwifery health services in Chile. Midwifery [Internet]. 2016;35:53-61. Available from: $h t t p: / / d x . d o i$. org/10.1016/j.midw.2016.01.018

20. Sadler M. Los hombres también se emocionan. Género y escenario del parto. Participación de hombres populares en el nacimiento de sus hijos e hijas. 2004;1-169. 
21. Maroto G, Castaño E, García M, Hidalgo N, Mateo I. Paternity and health services. Qualitative research on men's experiences during pregnancy, delivery and postpartum of theirs partners. Rev Esp Salud Publica. 2009;83(2):267-78.

22. Xue WL, Shorey S, Wang W, He HG. Fathers' involvement during pregnancy and childbirth: An integrative literature review. Midwifery [Internet]. 2018;62(March):135-45. Available from: https://doi. org/10.1016/j.midw.2018.04.013

23. Maroto-Navarro G, Pastor-Moreno G, Ocaña-Riola $R$, Benítez-Hidalgo $V$, García-Calvente Mdel M, Gutiérrez-Cuadra Mdel P, Gijón-Sánchez MT, del Río-Lozano M, Marcos-Marcos J. Male and female involvement in the birth and child-rearing process. J Clin Nurs. 2013 Nov;22(21-22):3071-83. doi: 10.1111/jocn.12153. Epub 2013 Sep 6. PMID: 24007478.

24. Organización Panamericana de la Salud. Declaración De Alma-Ata. Conf Int sobre Atención Primaria Salud, Alma-Ata, URSS, 6-12 septiembre 1978. 2012;1-3.

25. Carlos J, Guillermo L, Aguirre-garcía JC, Jaramillo-echeverri LG, Jaramillo-echeverri JC, Guillermo L. Aportes Del Método Fenomenológico a La Investigación Educativa. Rev Latinoam Estud Educ. 2012;8(2):51-74.

26. Hernandez, Fernandez y Baptista. Metodología de la investigación. Sexta edición. Mc Graw Hill. México. 2014.

27. Vázquez ML, Ferreira da Silva MR, Mogollón A, Fernández de Sammamed MJ, Delgado ME, Vargas I. Introducción a las técnicas cualitativas de investigación aplicadas en salud. Cursos GRAAL 5. Consorci Hospitalari de Catalunya. Universitat $\mathrm{Au}^{-}$ tónoma de Barcelona. Servei de Publicacions. Bellaterra. 2006.

28. Galeano ME. Estrategias de investigación social cualitativa. El giro en la mirada. La Carreta Editores E.U. Medellín. 2004

29. Krause M. La investigación cualitativa: Un campo de posibilidades y desafíos. Rev Temas Educ [Internet]. 1995; $\mathrm{N}^{\circ} 7$ (May):19-36. Available from: http://www.researchgatenet/ publication/215561167_La_investigacin_cualitativa_Un_campo_de_posibilidades_y_desafos/file/f6 57e40037e485815e526ee69689a88d.pdf

30. Díaz Herrera C. Investigación cualitativa y análisis de contenido temático. Orientación intelectual de revista Universum. Rev Gen Inf y Doc. 2018;28(1):119-42.
31. Flick U. El diseño de investigación cualitativa. Colección: investigación cualitativa. Ediciones Morata S.L. Madrid. 2015.

32. Observatorio de Violencia Obstétrica Chile. Resultados primera encuesta sobre el nacimiento en Chile. Obs Violencia Obs Chile [Internet]. 2018;(Junio). Available from: https://ovochile.cl/descarga-resultados-primera-encuesta-sobre-el-nacimiento-enchile/

33. Chile C de M. MINSAL detecta alza de cesáreas en red pública y alista norma para regularlas. [Internet]. 2018. Available from: https://colegiodematronas.cl/index.php/2017/06/27/minsal-detectaalza-de-cesareas-en-red-publica-y-alista-normapara-regularlas/

34. Angeja ACE, Washington AE, Vargas JE, Gomez R, Rojas I, Caughey AB. Chilean women's preferences regarding mode of delivery: Which do they prefer and why? BJOG An Int J Obstet Gynaecol. 2006;113(11):1253-8.

35. Sadler M, Leiva Rojas G, Perelló A, et al. Preferencia por vía de parto y razones de la operación cesárea en mujeres de la Región Metropolitana de Chile. Revista del Instituto de Salud Pública de Chile 2018; 2: 22-29.

36. Braide ASG, Brilhante AV, Arruda CN, Mendonça FAC, Caldas JMP, Nations MK, et al. Sou homem e pai sim! (Re)construindo a identidade masculina a partir da participação no parto. Rev Panam Salud Publica. 2018;42:e190. https://doi. org/10.26633/RPSP.2018.190

37. Bond J. Changing expectations: factors influencing paternal involvement in pregnancy and childbirth. Trends in Urology \& Men's Health. 2012. 3(5):23-26. Doi.org/10.1002/tre.285 
ANEXO 1. Matriz de operacionalización de variables

\begin{tabular}{|c|c|c|}
\hline Variable & Dimensión & Subdimensión \\
\hline \multirow[t]{2}{*}{$\begin{array}{l}\text { Motivación de los } \\
\text { progenitores en par- } \\
\text { ticipar del proceso } \\
\text { de parto. }\end{array}$} & $\begin{array}{l}\text { Nivel de motivación } \\
\text { y factores asociados, } \\
\text { previo al hecho y pre- } \\
\text { sente/visión de futuro. }\end{array}$ & $\begin{array}{l}\text { Deseo de participar (o no). } \\
\text { Percepción de su rol. } \\
\text { Origen de la motivación, razones, expectativas previas, aspectos que } \\
\text { la componen. } \\
\text { Valores personales. asociados. } \\
\text { Vínculo con la pareja y paternidad asociados. } \\
\text { Expectativas, beneficios percibidos, oportunidades previstas. } \\
\text { Aspectos negativos previstos. }\end{array}$ \\
\hline & $\begin{array}{l}\text { Aspectos que lo des- } \\
\text { motivan, o desmotiva- } \\
\text { rían. }\end{array}$ & $\begin{array}{l}\text { Aspectos propios subjetivos (tristeza, temor, percepción de partici- } \\
\text { pación de los hombres, entre otros). } \\
\text { Aspectos propios objetivos (trabajo, otros). } \\
\text { Aspectos del entorno (físico, social, cultural rol de género). }\end{array}$ \\
\hline \multirow{4}{*}{$\begin{array}{l}\text { Experiencias de par- } \\
\text { ticipación del padre } \\
\text { en el proceso de par- } \\
\text { to. }\end{array}$} & $\begin{array}{l}\text { Experiencia control } \\
\text { gestacional. }\end{array}$ & \multirow{4}{*}{$\begin{array}{l}\text { Conocimientos previos y fuente de información. } \\
\text { Posibilidad de participación, en qué participó, causas de no partici- } \\
\text { pación, facilitadores y obstaculizadores. } \\
\text { Vivencia, sentimientos, pensamientos. } \\
\text { Aspectos positivos y negativos de la vivencia. } \\
\text { Interacción con la madre y el recién nacido. } \\
\text { Interacción con la institucionalidad sanitaria y el personal de salud, } \\
\text { trato percibido, integración. }\end{array}$} \\
\hline & Experiencia preparto. & \\
\hline & Experiencia del parto. & \\
\hline & Experiencia puerperio. & \\
\hline \multirow[t]{4}{*}{$\begin{array}{l}\text { Propuestas para su } \\
\text { mayor incorporación } \\
\text { en el proceso, desde } \\
\text { la perspectiva de los } \\
\text { progenitores. }\end{array}$} & Información & $\begin{array}{l}\text { Tipo de información (contenidos, formatos, fuentes). } \\
\text { Momento de la entrega de información (previa durante, para des- } \\
\text { pués). }\end{array}$ \\
\hline & Participación. & $\begin{array}{l}\text { Presencia física, acompañamiento a la madre. } \\
\text { Participación en procedimientos. } \\
\text { Participación en decisiones. }\end{array}$ \\
\hline & Trato. & $\begin{array}{l}\text { Contención, empatía, tacto, sensibilidad para acoger sentimientos, } \\
\text { integración, facilitación. }\end{array}$ \\
\hline & Propuestas. & $\begin{array}{l}\text { Propuestas para mejorar el proceso, propuestas para aumentar la } \\
\text { participación. }\end{array}$ \\
\hline
\end{tabular}

